\title{
Median Filtering for Optic Disc Segmentation in Retinal Image
}

\author{
Ibnu Fiandono*1, Kartika Firdausy ${ }^{2}$ \\ 1,2Universitas Ahmad Dahlan \\ ibnufiandono@gmail.com¹, kartika@ee.uad.ac.id²
}

\begin{abstract}
One of diabetic complications is vision disturbance known as Diabetic Retinopathy (DR). Early detection becomes vital to prevent the development of $D R$ by routine examination of the patient's eyes. This study developed a software application that can automatically segment the image of the eye retina, expected to assist eye specialists in performing DR detection using computer aids. The system implementation uses Visual C\# 2010 software with 36 retinal images of the Drishti-GS1 dataset. The clinical features of the retina that can be used to detect DR are microaneurysms, hard and soft exudates, hemorrhages, neovascularization, and macular edema. Optic Disc (OD) segmentation is an important step to detect these features. This study aimed to detect $O D$ using red channel, inversion, contrast enhancement, median filtering, and thresholding. The final result of the $O D$ segmentation was validated $b$ y measuring the positive predictive value (PPV). The result of PPV for the detection of OD to ground truth image reached $90.924 \%$.
\end{abstract}

Keywords: Segmentation, Optic Disc, Diabetic Retinopathy, Median Filter, Retinal Fundus Image

\section{Introduction}

Diabetes can cause some complications exemplified by kidney failure, heart problems, and visual disturbance like diabetic retinopathy. Diabetic retinopathy (DR) is a problem in retinal blood vessels found in diabetics [1]. According to a publication issued by The Fred Hollow Foundation [2], one of three diabetic patients suffers from diabetic retinopathy. Every diabetic patient, ideally, has routine eye examination to have early detection on indicated vision disturbances. This examination employs fundus camera to capture retinal images.

Some symptoms on retinal abnormality caused by diabetic retinopathy are as follows [3]:

1. Microanerysms (MA), the initial symptom on retinal damages showing the existence of red dots having a size of less than $125 \mu \mathrm{m}$.

2. Hard Exudates, protein in white or yellowish color on retina's blood vessels with small dots, located on the outer layer of retina.

3. Soft Exudates, white wound.

4. Haemorrhages (HEM), uncommon form and reddish sized more than $125 \mu \mathrm{m}$.

5. Neovazcularisation (NV), abnormal growth in blood vessels on the surface of retina.

6. Macular Edema (ME), swelling retinal.

\section{Research Method}

Optic Disc (OD) segmentation is an important stage to detect these features. Optic Disc (OP) or eye nerve center is an area in which eye nerves entering retina and a center of all optic nerves [4]. The image objects used are 36 retinal images from dataset Drishti-GS1. They used PNG image format with resolution of $2896 \times 1944$ pixels. Moreover, this research employs Visual $\mathrm{C \#}$ 2010. The system design is presented in Figure 2. Preprocessing stage consists of channel extraction, invers, and contrast enhancement. Gaussian filtering and thresholding are utilized for segmentation.

\subsection{Preprocessing}

The image quality as the preprocessing results will significantly affect the segmentation results. Red channel is produced from the extraction of red component from retinal image using RGB format, as presented in Figure 2. Red channel was utilized in the output results due to its higher contrast level than that of other channels [4]. It was very helpful in the segmentation process in obtaining the Optic Disc Invers or the negation, an image processing method to reverse dark to bright colors [5][6]. The equation for invers operation utilized Equation 1.

Fiandono, I., \& Firdausy, K. (2018). Median Filtering for Optic Disc Segmentation in Retinal Image. Kinetik : Game Technology, Information System, Computer Network, Computing, Electronics, and Control, 3(1). http://dx.doi.org/10.22219/kinetik.v3i1.247

Paper submitted on July 04, 2017; Revision on July 27, 2017; Received September 06, 2017 


$$
K o=K \max -K i
$$

Kmax is the maximum intensity value of 255 for 8 bit image format. Moreover, Ko represents the color value output, and Ki represents the color value input. Contrast enhancement would be implemented to obtain inverted image. The elimination of background retinal image becomes necessary in order to acquire the expected output having sharper Optic Disc quality. Equation 2 is utilized to increase contrast [5].

$$
K o=G(K i-P)+P
$$

Contrast enhancement on fundus retinal image aims to ease Optic Disc segmentation process. The expected output of this contrast enhancement process aims to eliminate other unnecessary elements other than Optic Disc to achieve better identification.

\subsection{Segmentation}

Segmentation process plays the most important role in Optic Disc detection process on retinal image. The methods employed on this research were median filtering and thresholding.

Median filtering represents a mean value of a data set. The real pixel values are also calculated in the median computation. Median filtering has become significantly popular to handle some types of noise from due to its capability in providing better noise reduction and less blurred results. This median filtering process used $3 \times 3,5 \times 5,7 \times 7$, and $9 \times 9$ dimensions [7][8]. The result from median filtering is commonly called embroidery effect due to its resulting effects showing similar results with blurred crayon paintings. Median filtering can be calculated by the following Equation 3.

$$
f(x, y)=\text { median }\{g(s, t)\}
$$

Thresholding employs a conversion process of red channel image into binary image or black and white image; therefore, image areas related to object and background can be clearly recognized [9][10]. Developing new images can be processed using thresholding, defined using the following Equation 4.

$$
K o=\left\{\begin{array}{l}
1 \text { if } \mathrm{Ki}>\mathrm{T} \\
0 \text { if } \mathrm{Ki}<\mathrm{T}
\end{array}\right.
$$

\subsection{Evaluation}

The evaluation process was the last process from the segmentation of eye retinal image by validating the comparison results between retinal images from segmentation process with retinal images suffering from diabetic retinopathy (ground truth). This evaluation process was based on Positive Predictive Value (PPV) using the following Equation 5.

$$
P P V=\frac{T P}{T P+F P} \times 100 \%
$$

True Positive (TP) represents the number of Optic Disc pixels correctly detected; meanwhile, False Positive (FP) shows the number of non-Optic Disc pixels recognized.

\section{Research Result and Discussion}

This research utilized application form produced by utilizing $\mathrm{C \#} \mathrm{programming} \mathrm{language} \mathrm{on}$ Visual Studio 2010 Ultimate, depicted in Figure 3. The red channel extraction from original RGB image was the initial process implemented. Red channel, presented in Figure 4, was chosen because of its higher contrast value from blue and green channel. Moreover, the complement or inverting operation was conducted, followed by contrast enhancement as its results are presented in Figure 5 and Figure 6.

After acquiring image as the result of preprocessing, segmentation process would be implemented, initiated from median filtering (using $5 \times 5$ matrix) to thresholding process. The filtering process highly depends on contrast enhancement results, as more number of

KINETIK Vol. 3, No. 1, February 2018: 73-80 
unnecessary components eliminated leads to better filtering results. Conclusively, the segmentation process becomes a well-implemented operation. The result of median filter is presented by Figure 7 .

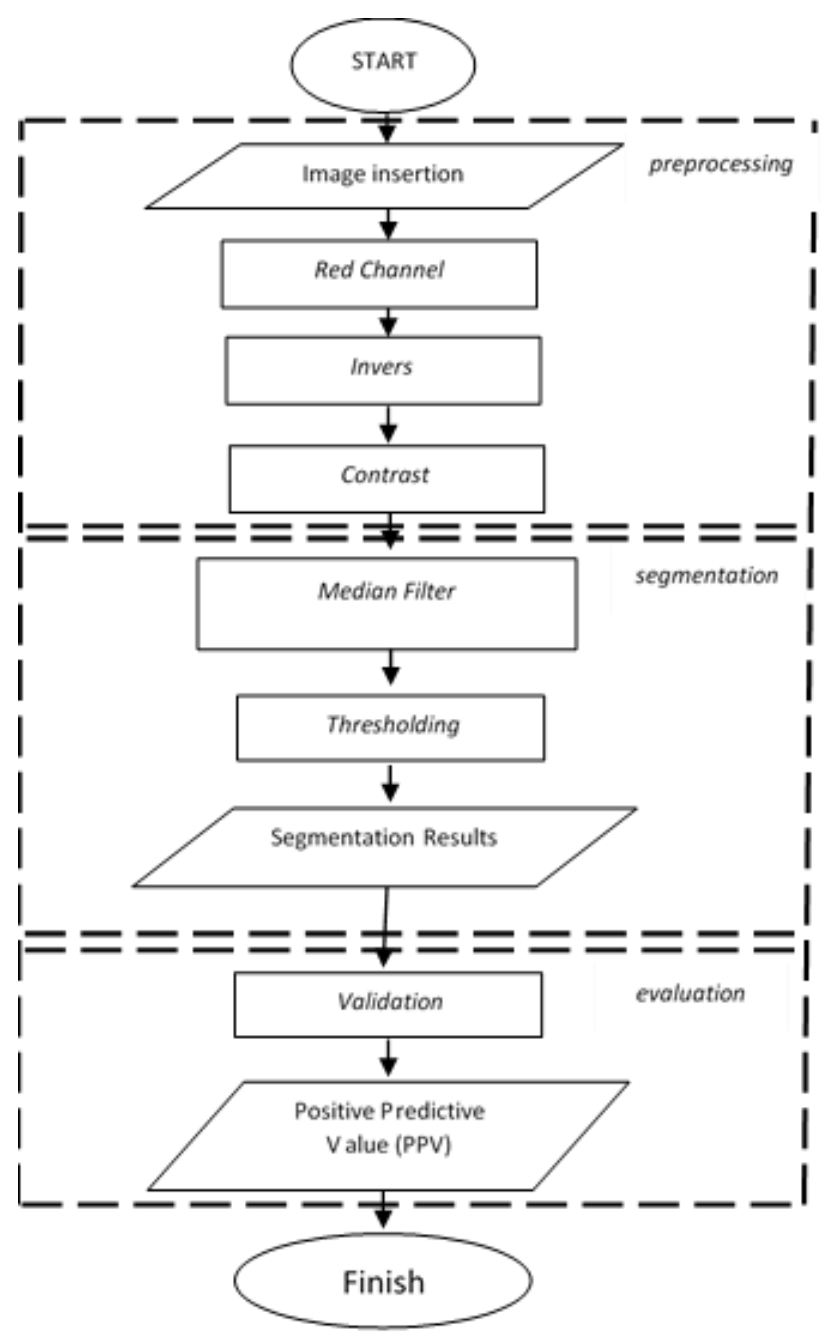

Figure 1. Research Method Flowchart

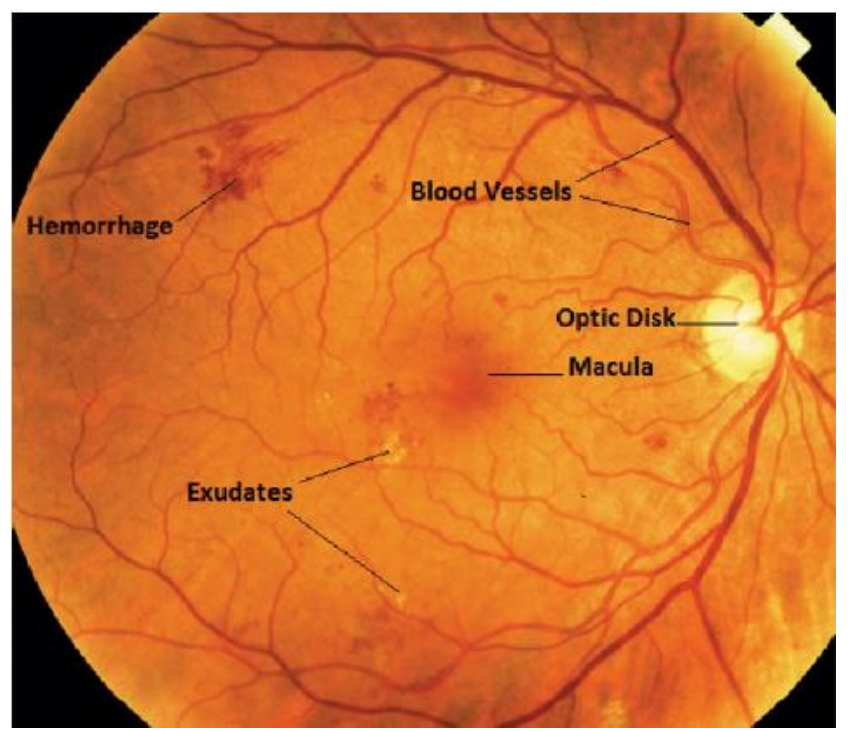

Figure 2. Retinal Fundus Image 


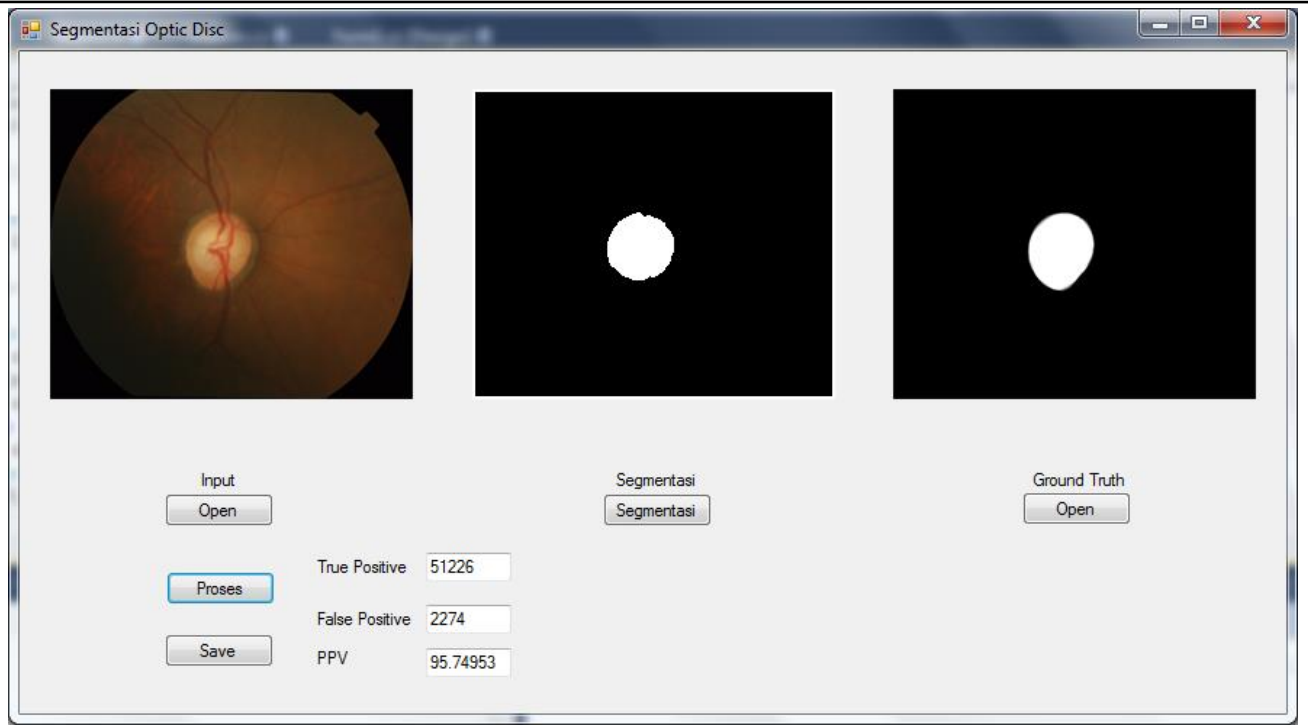

Figure 3. Application Form of OD Segmentation

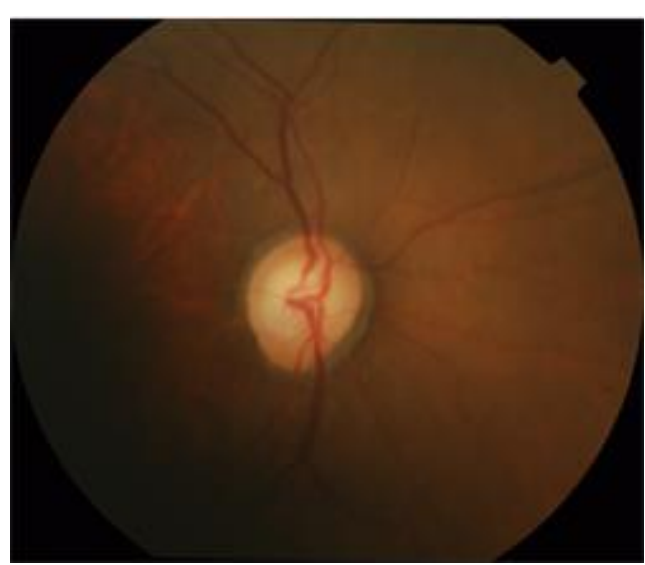

a

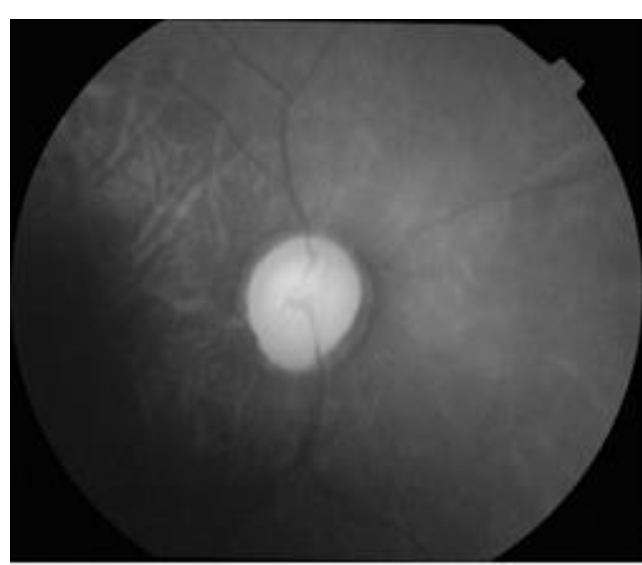

b

Figure 4. (a) Original Image (b) Red Channel

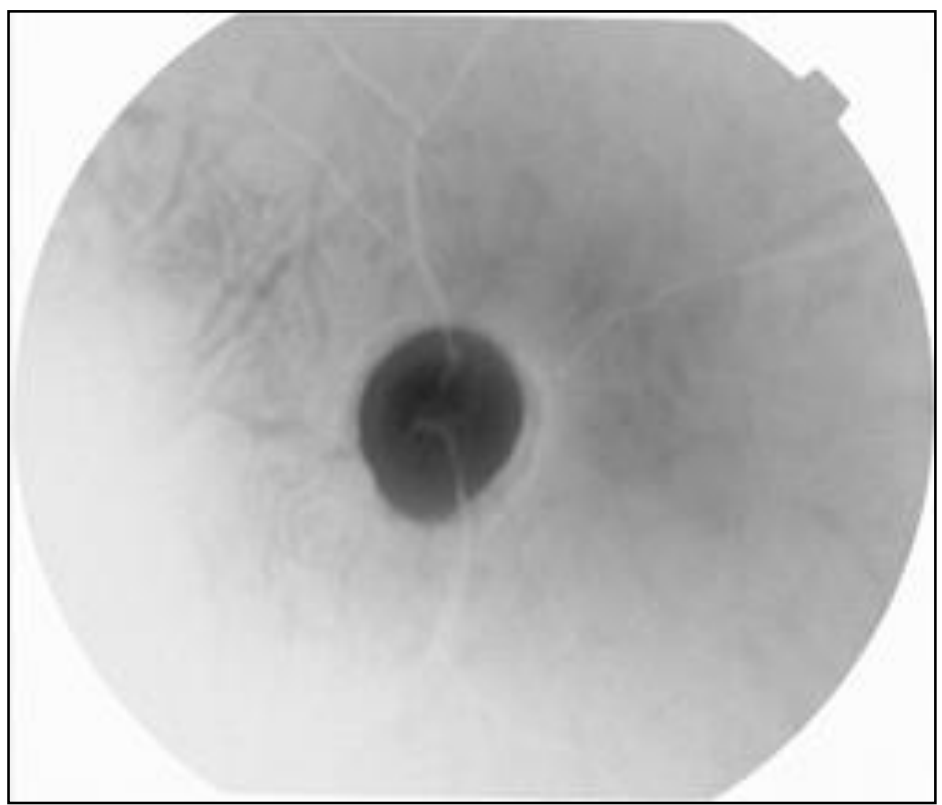

Figure 5. Inverted Image

KINETIK Vol. 3, No. 1, February 2018: 73-80 


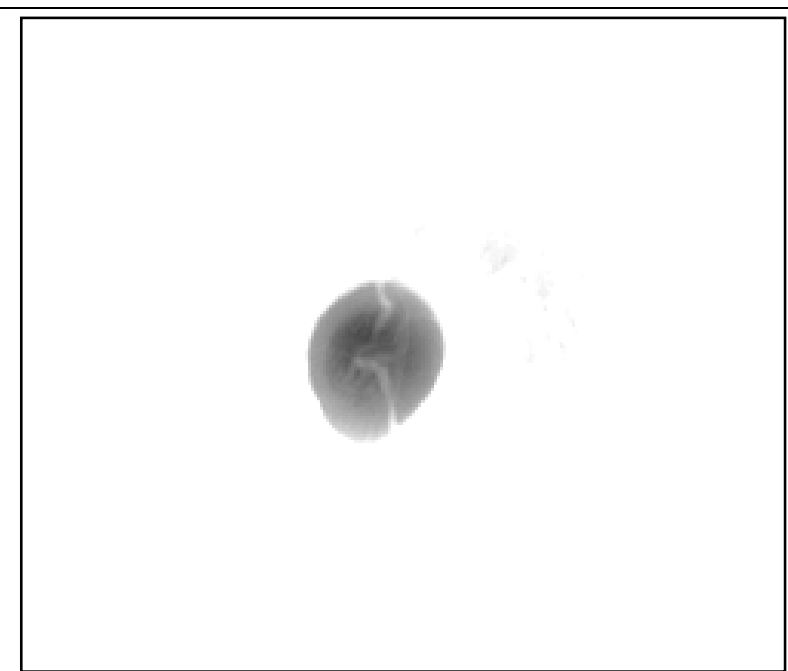

Figure 6. Image after Contrast Enhancement

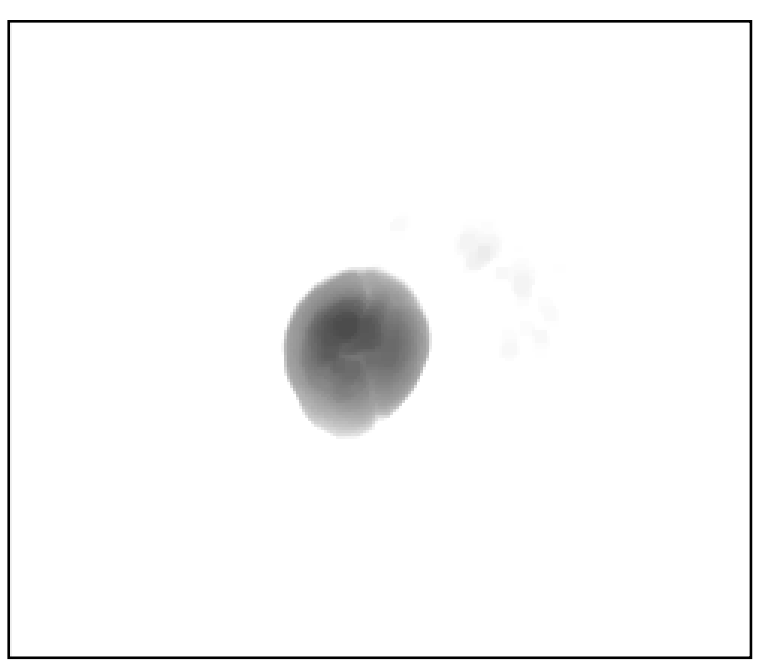

Figure 7. Image after Median Filter

The following process implemented thresholding process to obtain binary image. It utilized edge 170. Figure 8 presents the segmentation process and ground truth image.

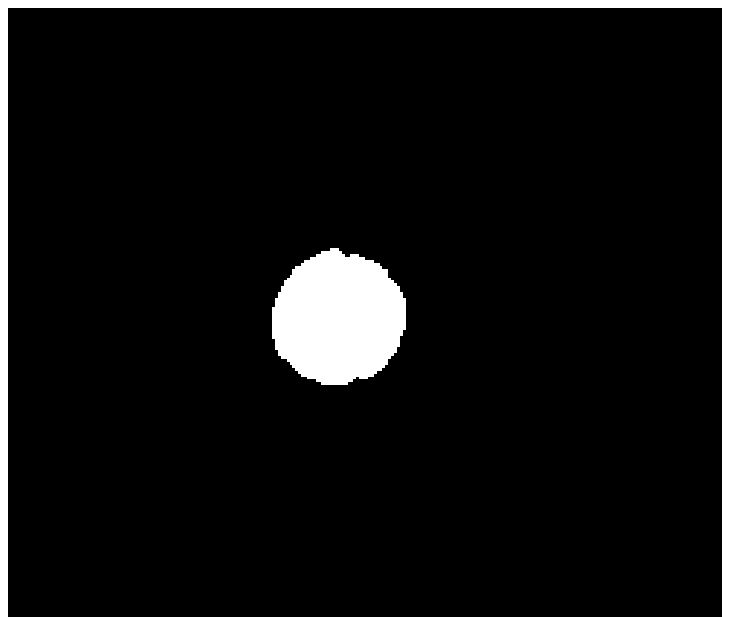

a

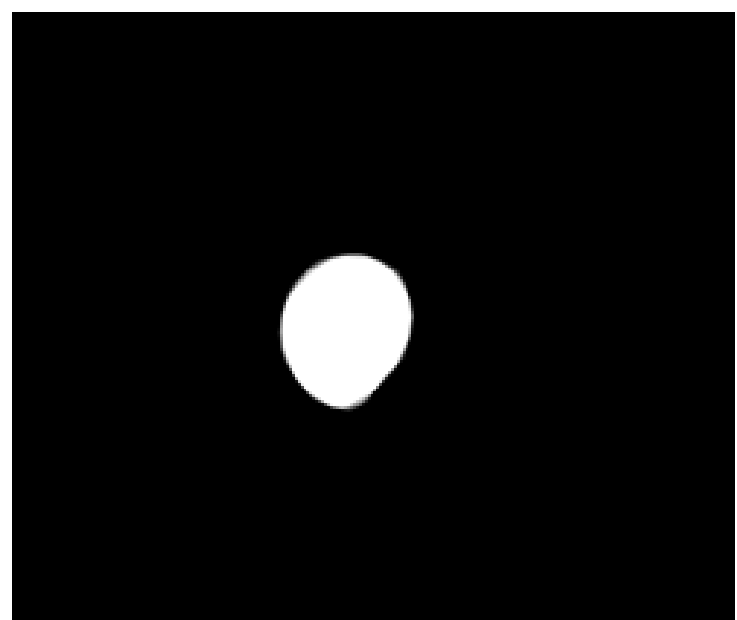

b

Figure 8. (a) Image after Segmentation (b) Ground Truth Image 
Furthermore, OD Image after segmentation process was compared with ground truth image from Drishthi-GS1. The result is presented by Table 1.

Table 1. PPV Values

\begin{tabular}{|c|c|c|c|}
\hline $\begin{array}{l}\text { Image } \\
\text { Number }\end{array}$ & TP & FP & PPV \\
\hline 1 & 48580 & 2670 & 94.79024 \\
\hline 2 & 50447 & 3053 & 94.29346 \\
\hline 3 & 50856 & 2644 & 95.05794 \\
\hline 4 & 48123 & 3127 & 93.89854 \\
\hline 5 & 50385 & 2865 & 94.61972 \\
\hline 6 & 47394 & 6106 & 88.58692 \\
\hline 7 & 49213 & 4037 & 92.41878 \\
\hline 8 & 50660 & 2590 & 95.13615 \\
\hline 9 & 49981 & 3269 & 93.86103 \\
\hline 10 & 50854 & 2396 & 95.50047 \\
\hline 11 & 51226 & 2274 & 95.74953 \\
\hline 12 & 51146 & 2604 & 95.15535 \\
\hline 13 & 42112 & 2388 & 94.63371 \\
\hline 14 & 47976 & 5524 & 89.67477 \\
\hline 15 & 472 & 6295 & 364 \\
\hline 16 & 50580 & 2920 & 94.54206 \\
\hline 17 & 51287 & 2213 & 95.86355 \\
\hline 18 & 50606 & 2894 & 94.59065 \\
\hline 19 & 51133 & 2367 & 95.5757 \\
\hline 20 & 50146 & 3354 & 93.73084 \\
\hline 21 & 48648 & 4602 & 91.35775 \\
\hline 22 & 50819 & 2431 & 95.43474 \\
\hline 23 & 50369 & 2881 & 94.58967 \\
\hline 24 & 50302 & 2948 & 94.46385 \\
\hline 25 & 47754 & 5496 & 89.67887 \\
\hline 26 & 51033 & 2217 & 95.83662 \\
\hline 27 & 51162 & 2338 & 95.62991 \\
\hline 28 & 51482 & 2268 & 95.78047 \\
\hline 29 & 50524 & 2976 & 94.43738 \\
\hline 30 & 51611 & 2139 & 96.02047 \\
\hline 31 & 50974 & 2526 & 95.2785 \\
\hline 32 & 39180 & 5220 & 88.24324 \\
\hline 33 & 46628 & 6872 & 87.15514 \\
\hline 34 & 51284 & 2216 & 95.85794 \\
\hline 35 & 50987 & 2263 & 95.75023 \\
\hline 36 & 49452 & 4048 & 92.43364 \\
\hline Average & 49503.3 & 3306 & 93.71837 \\
\hline
\end{tabular}

This comparison is based on Positive Predictive Value (PPV) equation. The obtained PPV results depend on True Positive (TP) values and False Positive (FP) values showing average score of $90.924 \%$.

KINETIK Vol. 3, No. 1, February 2018: 73-80 


\section{Conclusion}

The retinal image segmentation using median filter method has been completed resulting the average PPV value of $93.71837 \%$.

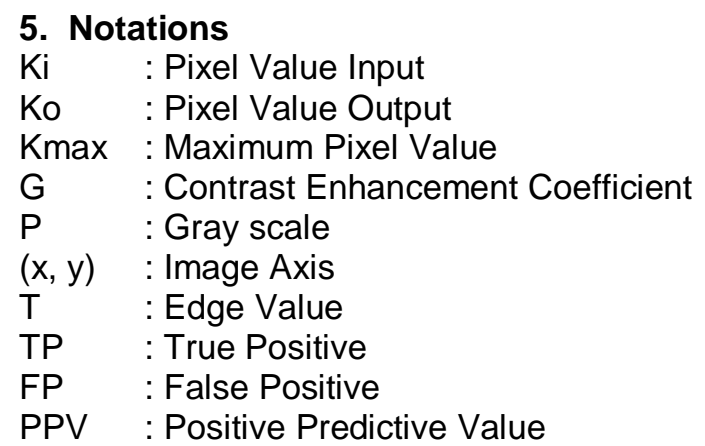

\section{References}

[1] SNEC, "Diabetic Retinophaty," $2015 . \quad$ Retrieved from http://www.snec.com.sg/about/international/menuutama/kondisimataandperawatan/commo n-problems/Pages/Diabetic-Retinopathy.aspx

[2] Hollow, F., "Diabetic Eye Health: A Guide for Health Professionals," 2015. Retrieved from http://www.idf.org/sites/default/files/IDF\%20EYE\%20Health\%20Care\%20Guide_INSIDE_FI N_WEB.pdf

[3] M. A. Ulinuha, I. K. E. Purnama, and M. Hariadi, "Optic Disc Segmentation on Diabetic Retinophaty Patients using GVF Snake," ITS Library, 2010.

[4] K. Firdausy and W.Oktoeberza, "Segmentation of Optic Disc using Dispersive Phase Stretch Transformation," International Annual of Engineering Seminar, 2016.

[5] B. Achmad and K. Firdausy, "Digital Image Processing using DELPHI," Yogyakarta: Penerbit Andi, 2013.

[6] R. E. Putra, H. Tjandrasa, and N. Suciati, "Review Algoritma Segmentasi Pembuluh Darah Pada Citra Fundus Retina Mata Untuk Membantu Diagnosis Diabetic Retinopathy", JUTI, Vol. 10, No. 2, 2012.

[7] Sutoyo, T. et al., "Theory of Digital Image Processing," Yogyakarta: Andi Offset, 2009.

[8] Dimitrov, D. et al., ,"Fundamentals of Computer Programming with C\# (The Bulgarian C\# Programming Book)," Sofia: Svetlin Nakov and Co., 2013.

[9] Kurnia, M.R., Tjandrasa, H. and Wijaya, Y., "Implementation of Segmentation on Retinal Blood Vessel on Fundus Image using Texture, Thresholding, and Morphology operation," Engineering Journal of ITS Students' Online Scientific Publication, 1 (1), Pp. 1-6, 2012.

[10] Sitompul, R., "Diabetic Retinophaty," Journal of the Indonesian Medical Association, Vol 61, No 8, Pp. 337-341, 2011. 
